Research Article

\title{
Expression Patterns and Prognostic Values of ORMDL1 in Different Cancers
}

\author{
Tengjiao Zhu, Yingtong Chen, Shuyuan Min, Fang Li $(\mathbb{D}$, and Yun Tian
}

Peking University Third Hospital, 49 Huayuan North Road, Beijing 100191, China

Correspondence should be addressed to Fang Li; lf@bjmu.edu.cn and Yun Tian; tiany@bjmu.edu.cn

Received 6 February 2020; Revised 3 August 2020; Accepted 15 September 2020; Published 21 October 2020

Academic Editor: Maxim Golovkin

Copyright (C) 2020 Tengjiao Zhu et al. This is an open access article distributed under the Creative Commons Attribution License, which permits unrestricted use, distribution, and reproduction in any medium, provided the original work is properly cited.

\begin{abstract}
The mammalian orosomucoid-like gene family (ORMDL), containing ORMDL1, ORMDL2, and ORMDL3, is the important regulator of sphingolipid metabolism, which is relevant to cell growth, proliferation, migration, and invasion. Since the role of $O R M D L 1$ in cancers remained unclear, the main purpose of our study was to explore the expression patterns and prognostic values of ORMDL1 in different tumors, especially in cholangiocarcinoma (CHOL), lymphoid neoplasm diffuse large $\mathrm{B}$ cell lymphoma (DLBCL), acute myeloid leukemia (LAML), and thymoma (THYM). Bioinformatics tools including GEPIA, CCLE, LinkedOmics, cBioPortal, and TIMER databases were used. As a result, the expression levels of ORMDL1 in tumor tissues and normal tissues varied in different cancers, especially significantly upregulated in CHOL, DLBCL, LAML, and THYM. Moreover, ORMDL1 mRNA was also highly expressed in cell lines of DLBCL and LAML. Further studies showed that ORMDL1 overexpression was associated with poor prognosis in DLBCL, but not significant in CHOL, LAML, and THYM. Consistently, there were genetic alterations of ORMDL1 in DLBCL, and patients with genetic alterations indicated worse survival. Coexpressed genes and related biological events with ORMDL1 in DLBCL were found via LinkedOmics, Gene Ontology (GO), and Kyoto Encyclopedia of Genes and Genomes (KEGG) analysis. The relationship between ORMDL1 and cancer immune cells was investigated, and ORMDL1 expression was positively correlated with infiltrating levels of B cells. In conclusion, ORMDL1 is suggested to be a tumorigenic factor and considered as the potential therapeutic target and prognostic biomarker in DLBCL.
\end{abstract}

\section{Introduction}

The mammalian orosomucoid-like gene family (ORMDL), including ORMDL1, ORMDL2, and ORMDL3, encodes transmembrane proteins localized to the endoplasmic reticulum [1-5]. ORMDLs are primarily involved in negative feedback regulation of sphingolipid metabolism, ceramide synthesis, and unfolded protein response [3-10]. The fulllength human ORMDL1 cDNA was originally obtained after screening a human retinal cDNA library and confirmed to be located at chromosome 2q32 [1]. During the process of sphingolipid biosynthesis, serine palmitoyltransferase (SPT) catalyzed the critical rate-limiting step. Inhibition of ORMDL1 led to enhanced SPT activity and increased sphingolipid levels [6]. In addition, the expression levels of ORMDL1 were demonstrated to be significantly correlated with familial Alzheimer's disease-related presenilin (PS) mutations, manifesting as elevated ORMDL1 and ORMDL2 levels due to PS deficiency. Silencing of ORMDLs suppressed nicastrin maturation and $\gamma$-secretase function [2]. For ORMDL3, it was closely associated with asthma risk in childhood [11, 12] and participated in cellular stress response [13], lymphocyte activation [14], and eosinophil trafficking [15].

As an important component of the cell membrane, sphingolipids can regulate cell growth, proliferation, migration, invasion, and metastasis through cancer signaling pathways, in addition to exerting barrier function and maintaining membrane fluidity [16-18]. Since ORMDL1 was a regulator of sphingolipid levels in cells, we hypothesized that ORMDL1 might play a role in the pathogenesis and progression of tumors. Thus, our study shed light on the specific role of ORMDL1 in different tumors via bioinformatics analysis, particularly in cholangiocarcinoma (CHOL), lymphoid neoplasm diffuse large B cell lymphoma (DLBCL), acute myeloid leukemia (LAML), and thymoma (THYM). 


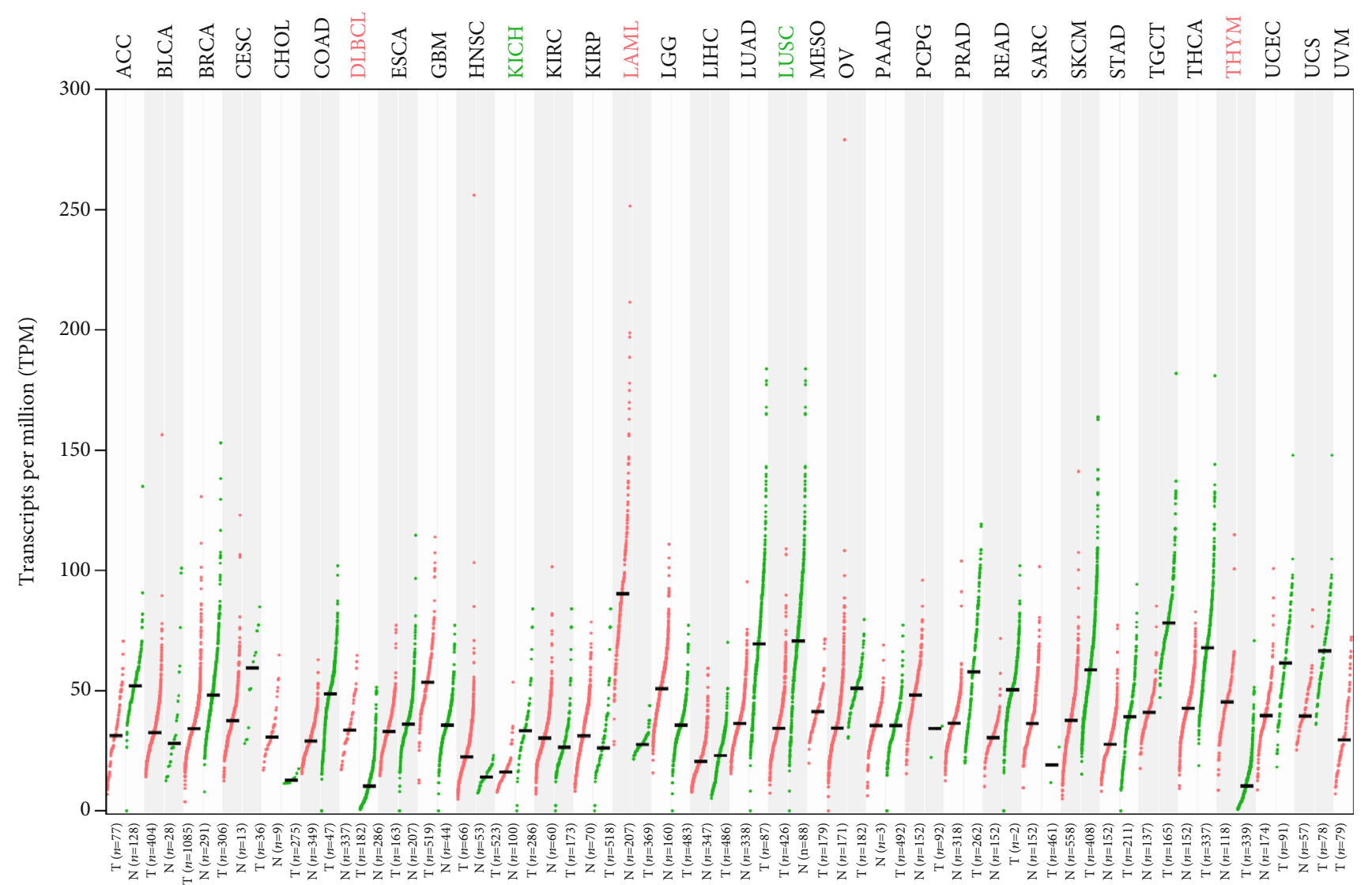

(a)

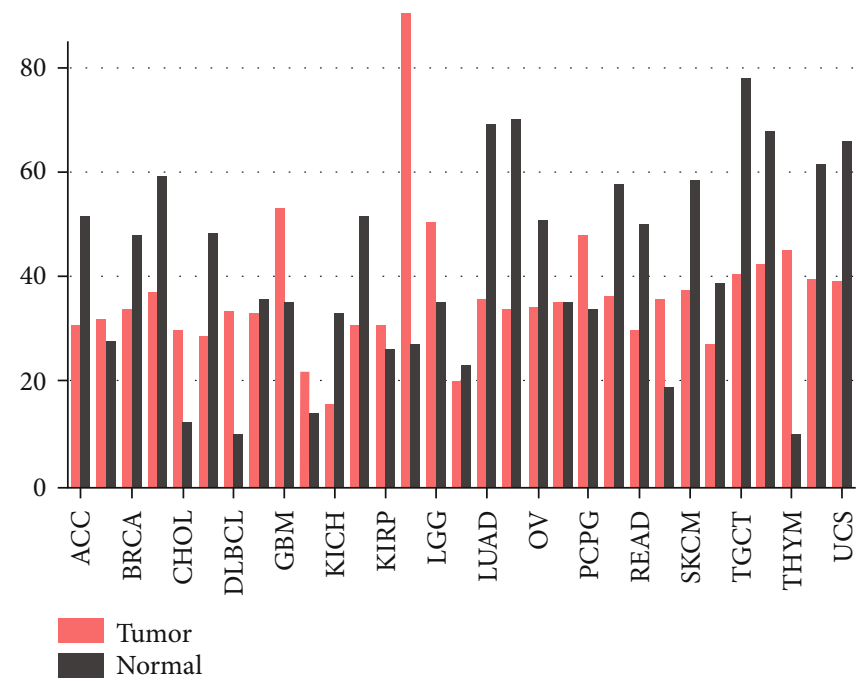

(b)

FIgURE 1: Continued 

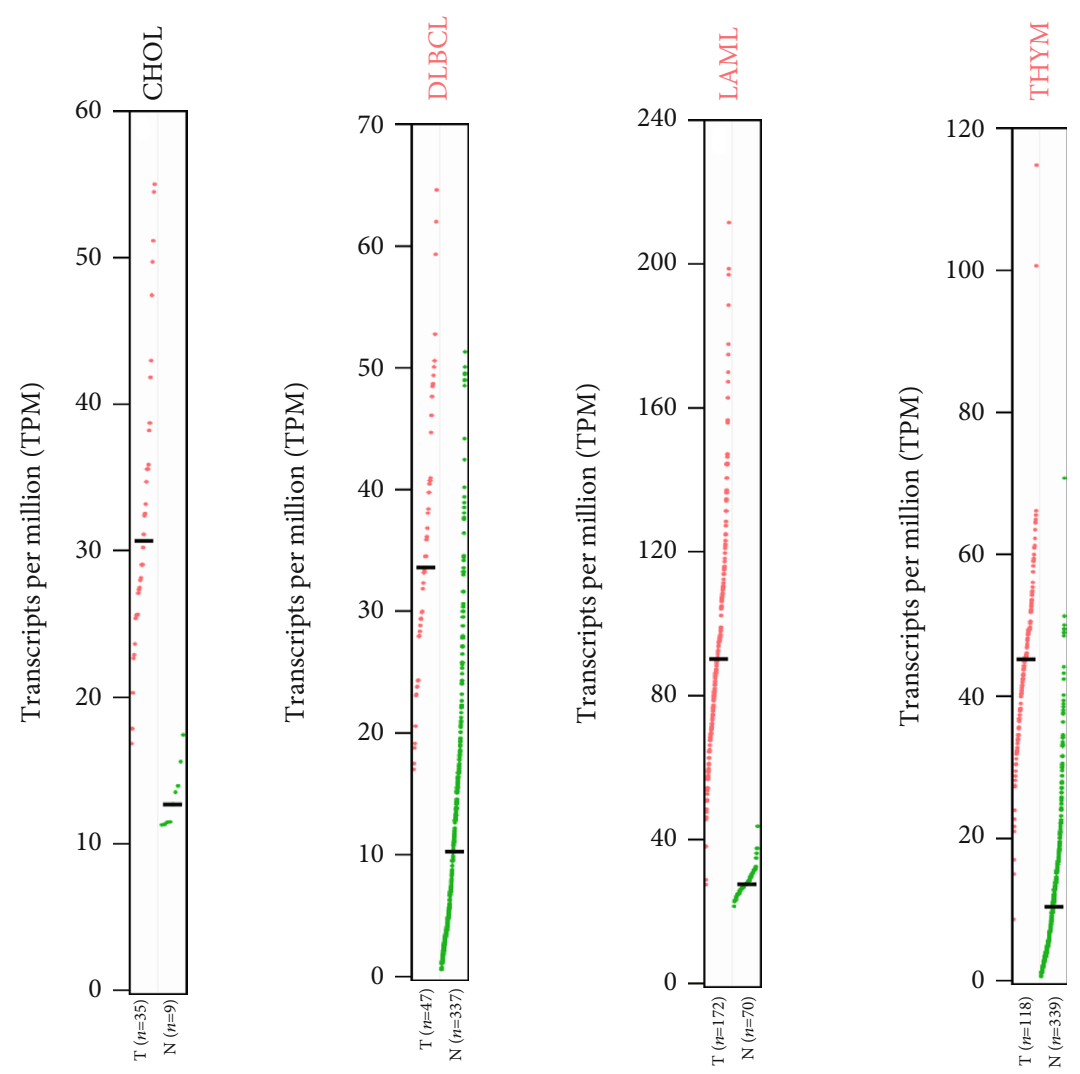

(c)

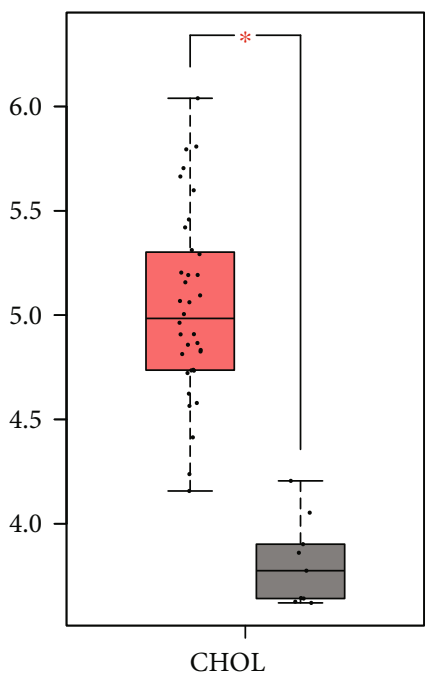

$(\operatorname{num}(\mathrm{T})=36 ; \operatorname{num}(\mathrm{N})=9)$

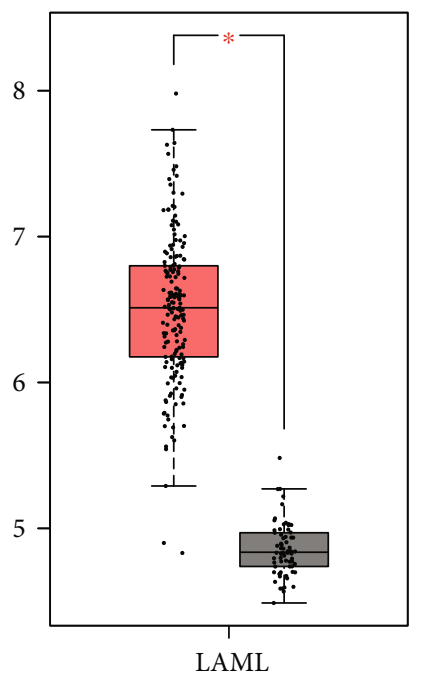

$(\operatorname{num}(\mathrm{T})=173 ; \operatorname{num}(\mathrm{N})=70)$

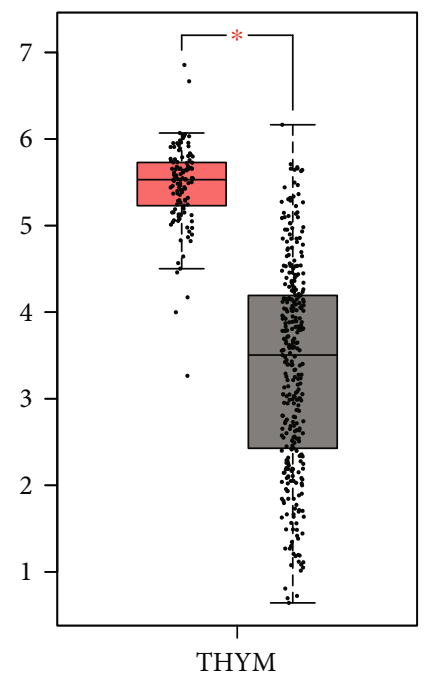

$(\operatorname{num}(\mathrm{T})=118 ; \operatorname{num}(\mathrm{N})=339)$

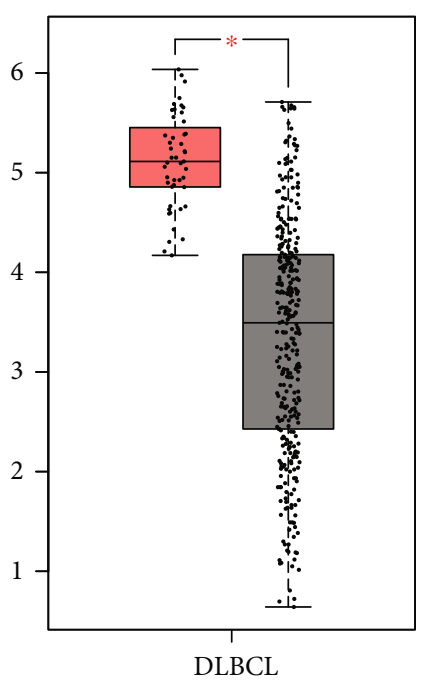

$(\operatorname{num}(\mathrm{T})=47 ; \operatorname{num}(\mathrm{N})=337)$

(d)

Figure 1: The expression levels of ORMDL1 in CHOL, DLBCL, LAML, and THYM (GEPIA). (a, b) The expression levels of ORMDL1 in pancancer. (c, d) The expression levels of ORMDL1 in CHOL, DLBCL, LAML, and THYM. ${ }^{*} P<0.05$. CHOL: cholangiocarcinoma; DLBCL: lymphoid neoplasm diffuse large B cell lymphoma; LAML: acute myeloid leukemia; THYM: thymoma.

We emphatically investigated the expression level of ORMDL1 in different types of cancers, the effect of ORMDL1 expression on patient prognosis, and the genetic alterations and the potential interaction of ORMDL1 with related genes especially in DLBCL.

\section{Methods}

2.1. GEPIA Dataset Analysis. Gene Expression Profiling Interactive Analysis (GEPIA, http://gepia.cancer-pku.cn/ index.html) is an online database. As an interactive web, 
mRNA expression (RNAseq): ORMDL 1

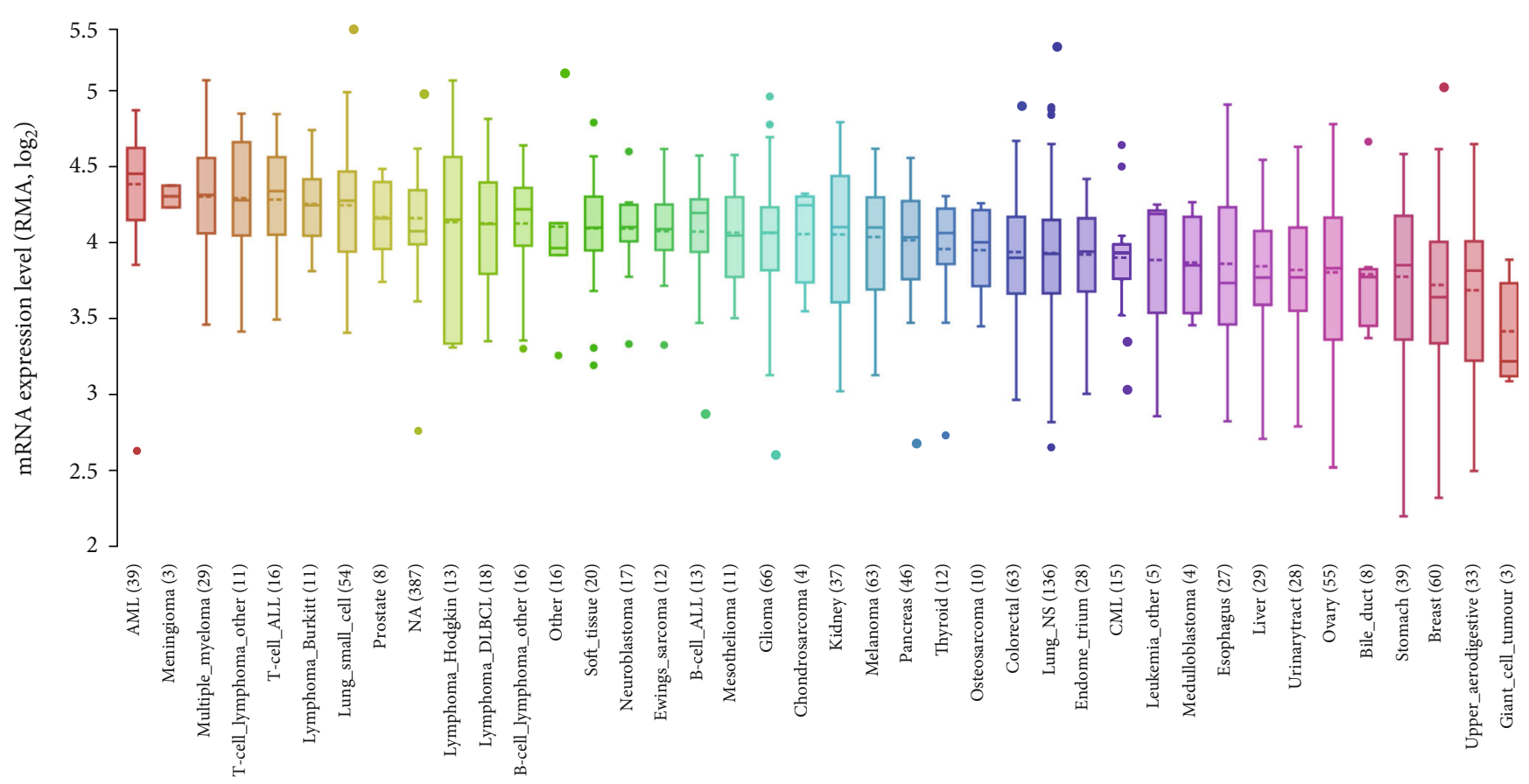

FIgURE 2: The expression of ORMDL1 in cell lines of different cancer types (CCLE).

GEPIA provides 9,736 tumors and 8,587 normal samples from The Cancer Genome Atlas (TCGA) and the Genotype-Tissue Expression (GTEx) projects for analyzing the RNA sequencing expression data [19]. GEPIA was used to analyze the differential expression of ORMDL1 between normal tissues and tumor tissues in 33 different types of cancer. We compared the expression patterns of ORMDL1 in four cancer types, including CHOL, DLBCL, LAML, and THYM. Moreover, GEPIA also provided the function for prognostic curve analysis and pathological stage evaluation.

2.2. CCLE Dataset Analysis. The Cancer Cell Line Encyclopedia (CCLE, http://www.broadinstitute.org/ccle/home) project is a collaboration between the Broad Institute and the Novartis Institutes for Biomedical Research and its Genomics Institute of the Novartis Research Foundation. It can be applied to conduct a detailed genetic and pharmacologic characterization of a large panel of human cancer models, develop integrated computational analyses that link distinct pharmacologic vulnerabilities to genomic patterns, and translate cell line integrative genomics into cancer patient stratification. CCLE provides public access to genomic data, analysis, and visualization for about 1,000 cell lines [20]. The expression of ORMDL1 was verified by the CCLE dataset.

2.3. LinkedOmics Dataset Analysis. LinkedOmics (http:// www.linkedomics.org/login.php) provides a newly developed platform for analyzing large-scale cancer omics data from TCGA and Clinical Proteomic Tumor Analysis Consortium
(CPTAC) [21]. We used LinkedOmics to inquire into the prognostic values of ORMDL1 expression in the four cancer types, including CHOL, DLBCL, LAML, and THYM. The survival differences were visualized by Kaplan-Meier plots. Furthermore, the correlation coefficient and coexpressed gene patterns were calculated according to the online instruction.

2.4. cBioPortal Analysis. The cBioPortal database (http:// cbioportal.org) is an online database that converts complex cancer genomic data from TCGA into well-understood genetic, epigenetic, and proteomic data, including somatic mutations, altered copy number, mRNA and miRNA expression, DNA methylation, and protein abundance data. It can be used to explore genetic changes in tumor samples and compare the effects of these changes on patient survival [22]. In our study, 48 DLBCL samples (TCGA, Provisional) with pathological reports were selected for further analysis of ORMDL1 genetic alterations using cBioPortal. The mutation plots were drawn to directly reflect all types of ORMDL1 genetic alterations. Additionally, Kaplan-Meier survival curves were constructed to analyze the influence of ORMDL1 genetic alterations on the DLBCL patient survival.

2.5. TIMER Analysis. Tumor Immune Estimation Resource (TIMER) is a comprehensive database for systematical analysis of the abundances of six immune infiltrates (B cells, CD4 + T cells, CD8+ T cells, neutrophils, macrophages, and dendritic cells) in diverse cancer types. The function of the gene module is to explore the correlation between gene expression 


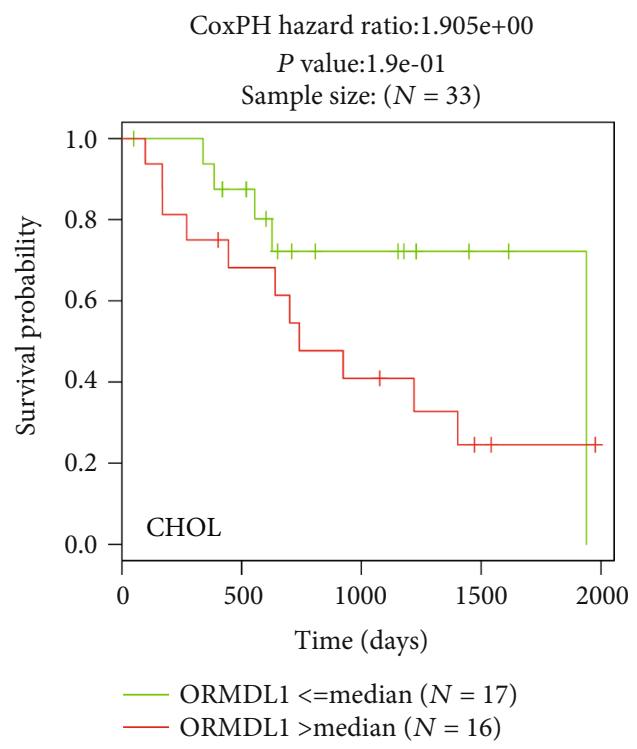

(a)

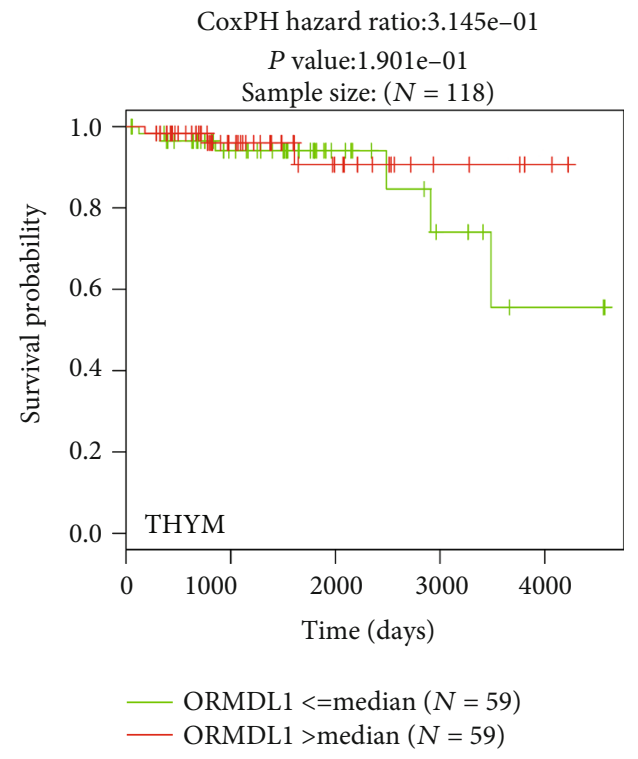

(c)

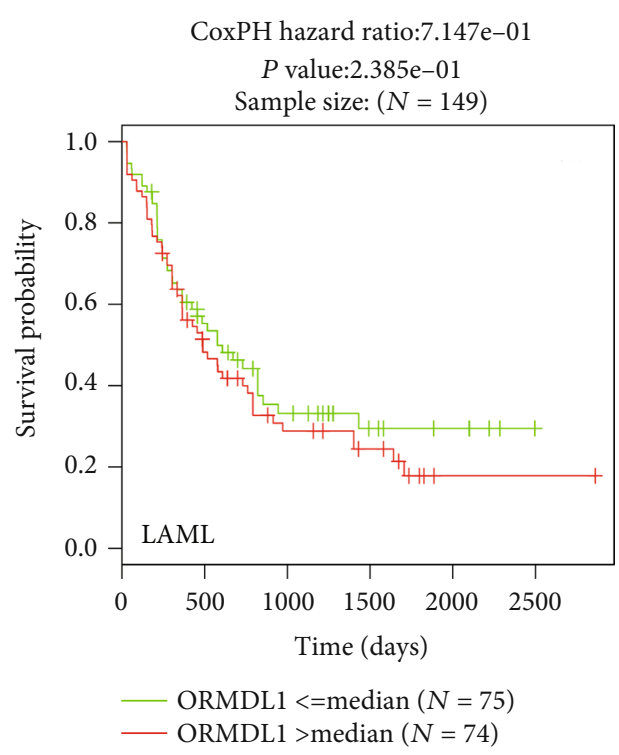

(b)

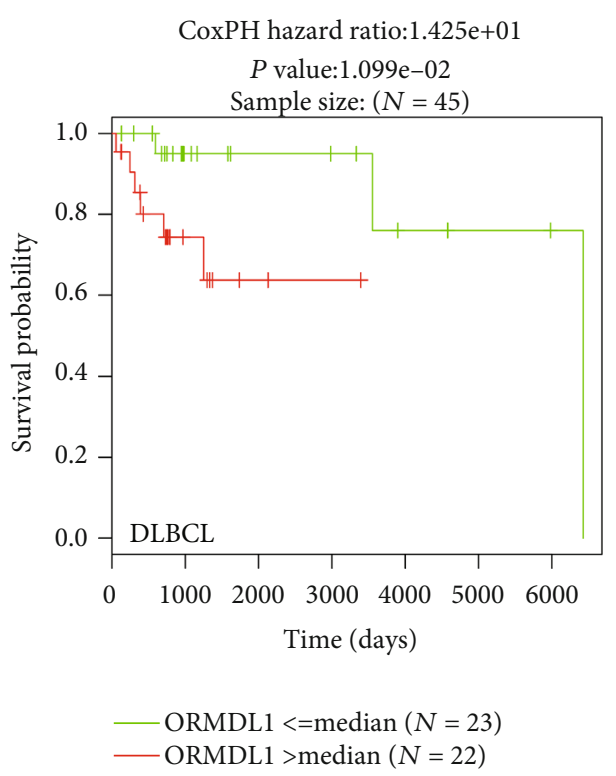

(d)

Figure 3: Continued. 


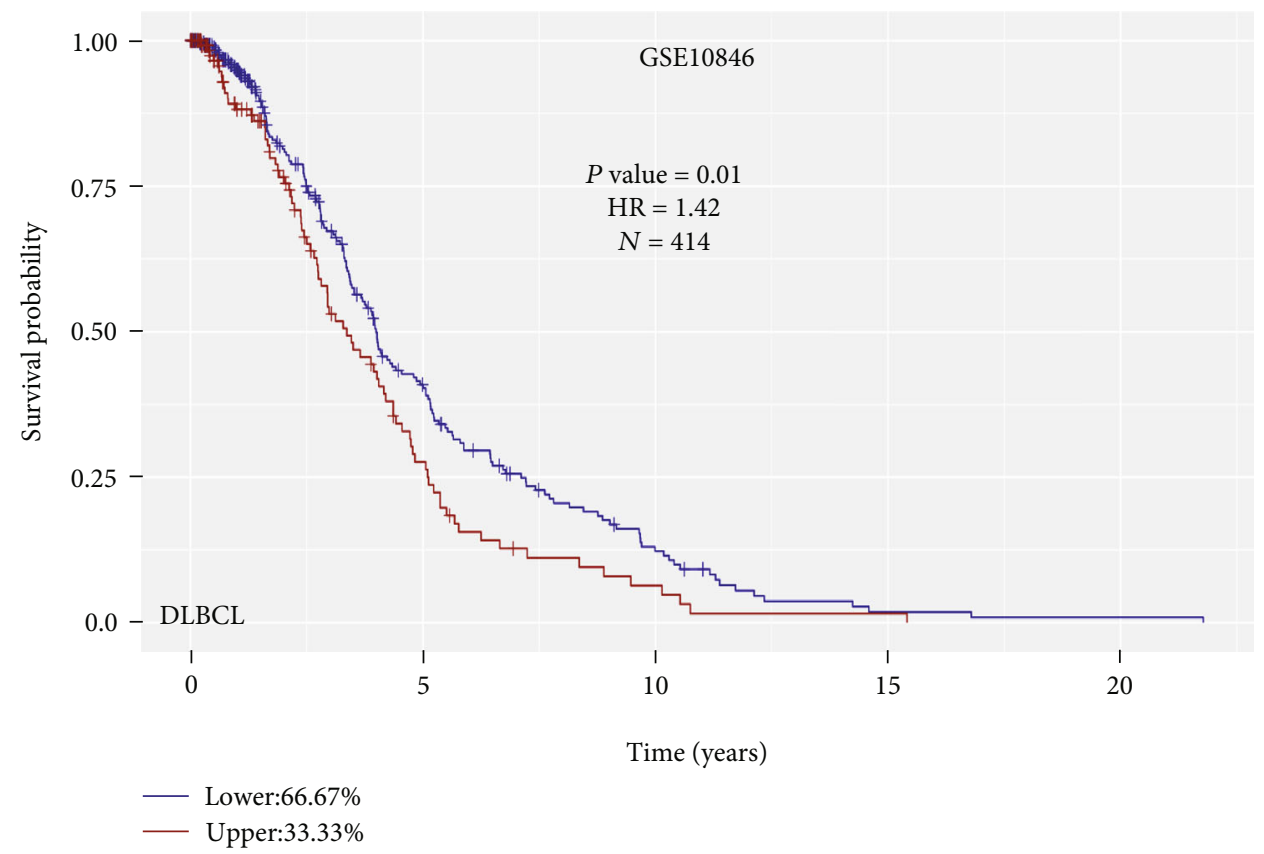

(e)

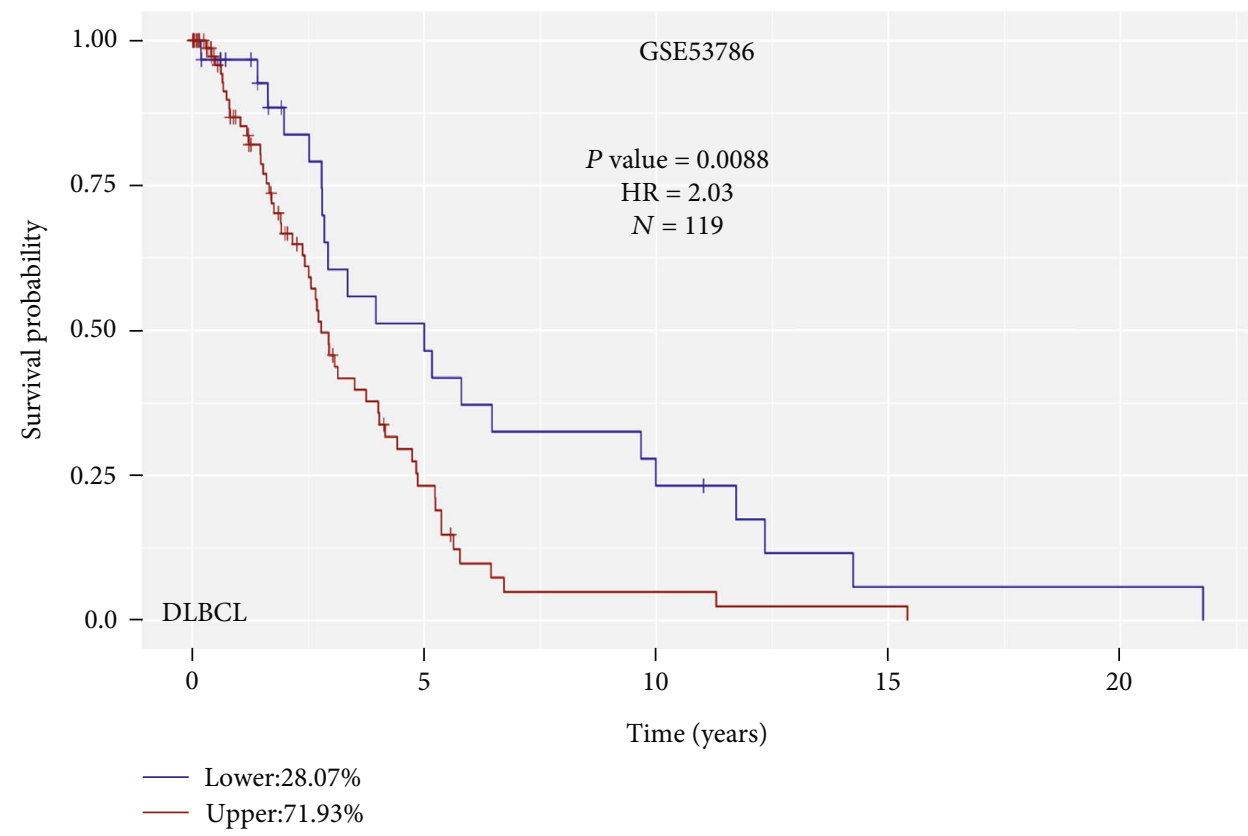

(f)

Figure 3: The prognostic value comparing the high and low expression of ORMDL1 in CHOL, LAML, THYM, and DLBCL (LinkedOmics and shinyGEO). (a-d) Overall survival curves of CHOL, LAML, THYM, and DLBCL, analyzed by LinkedOmics. (e, f) Overall survival curves of DLBCL, analyzed by shinyGEO. CHOL: cholangiocarcinoma; LAML: acute myeloid leukemia; THYM: thymoma; DLBCL: lymphoid neoplasm diffuse large B cell lymphoma.

and abundance of immune infiltrates [23]. In this study, the relationship between ORMDL1 expression and the six immune cells was estimated by TIMER in DLBCL.

2.6. Statistical Analysis. The difference in ORMDL1 expression between tumor tissues and normal tissues was compared with an independent $t$-test. ORMDL1 expression in different clinical stages was evaluated using one-way ANOVA. The relationship between ORMDL1 expression and patient prognosis was detected using the KaplanMeier survival analysis and log-rank test. The correlation between ORMDL1 and related genes was analyzed using the Pearson correlation test. $P<0.05$ indicated statistical significance. 


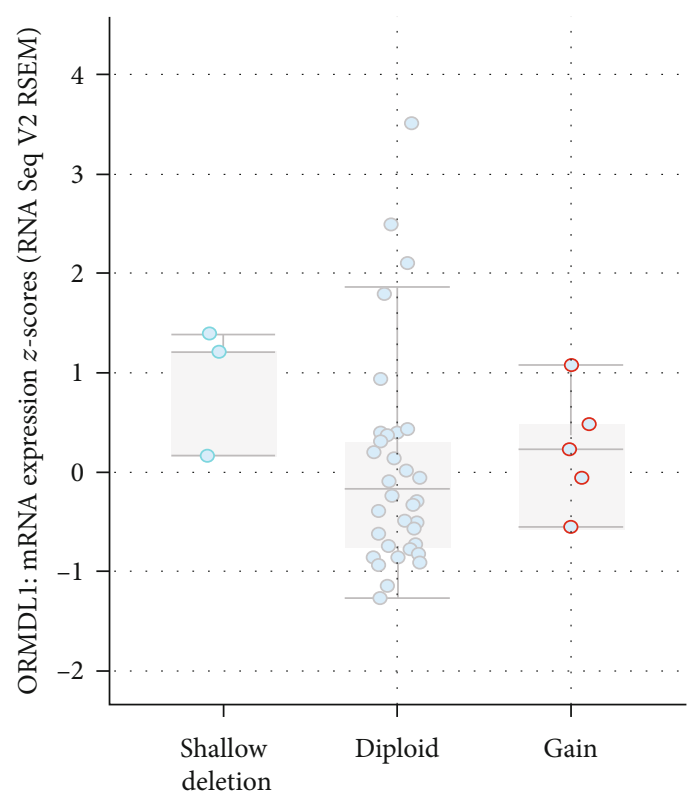

ORMDL1: putative copy-number alterations from GISTIC

$$
\begin{array}{ll}
\text { Not mutated } & \text { Diploid } \\
\circ \text { Gain } & \text { Shallow deletion }
\end{array}
$$

(a)

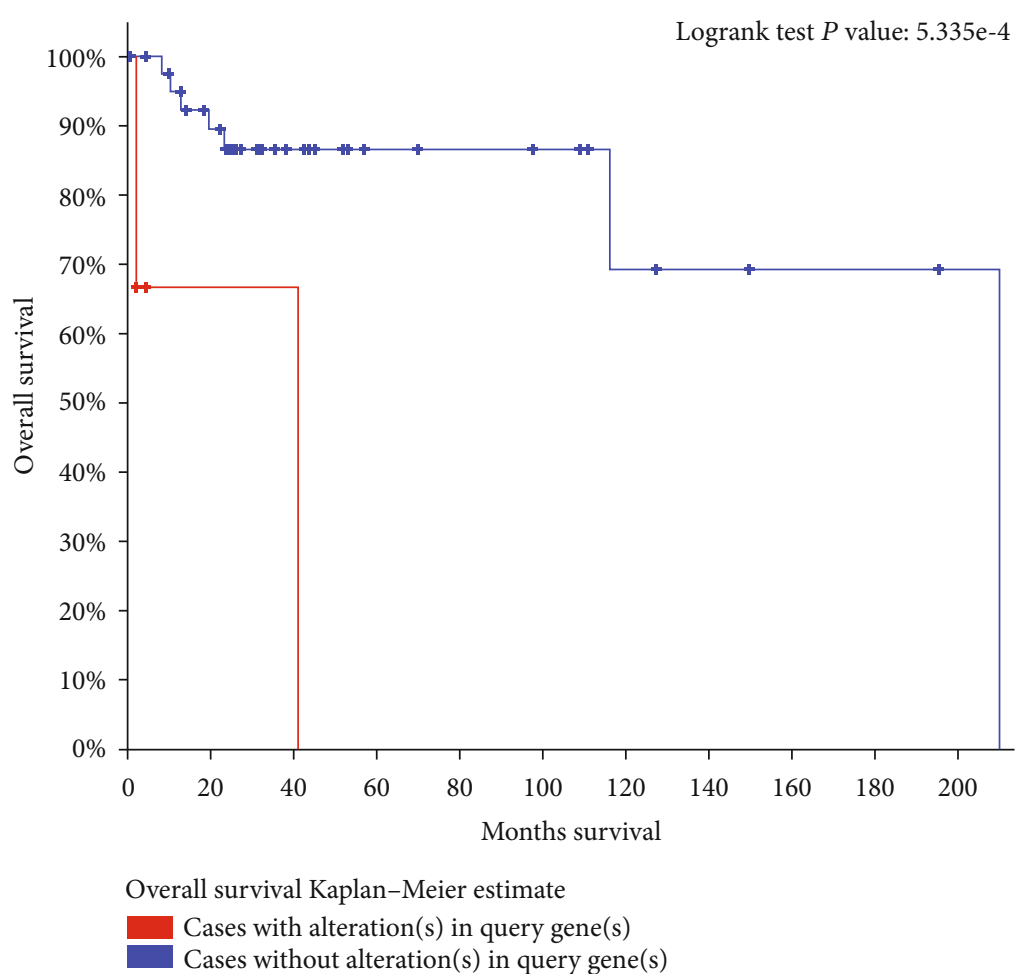

(b)

FIGURE 4: Genetic alterations of ORMDL1 in DLBCL (cBioPortal). (a) ORMDL1 mutation analysis in DLBCL. (b) Overall survival curves with or without ORMDL1 alterations in DLBCL. DLBCL: lymphoid neoplasm diffuse large B cell lymphoma.

\section{Results}

3.1. Expression Levels of ORMDL1 in Different Types of Human Cancers. To determine differences of ORMDL1 expression between tumor samples and normal samples, the ORMDL1 mRNA levels of different tumor samples and normal samples were analyzed using GEPIA. The differential expression of ORMDL1 in tumor samples and normal samples from all TCGA cancer types is listed in Figures 1(a) and 1(b). In particular, the results indicated that ORMDL1 expression levels were significantly upregulated in $\mathrm{CHOL}$, DLBCL, LAML, and THYM compared to their corresponding normal tissues (Figures 1(c) and 1(d)).

3.2. ORMDL1 mRNA Expression in Different Kinds of Cancer Cell Lines. By collecting genetic information from CCLE, investigation of ORMDL1 expression was extended to various cancer cell lines. As a result, ORMDL1 mRNA was found to be highly expressed in cell lines of LAML and DLBCL, which ranked $1^{\text {st }}$ and $11^{\text {th }}$ among 40 kinds of cancers (Figure 2).

3.3. The Prognostic Influence of ORMDL1 on CHOL, DLBCL, $L A M L$, and THYM. We further explored the influence of ORMDL1 expression levels on the survival of patients in four cancer types, including CHOL, DLBCL, LAML, and THYM. The Kaplan-Meier curves and log-rank test analysis revealed that increased ORMDL1 was associated with poor overall survival (OS) in DLBCL significantly, but not in CHOL, LAML, and THYM by LinkedOmics (Figures 3(a)-3(d)).
Also, similar results were predicted in GSE10846 and GSE53786 using shinyGEO online tool, which could analyze patient survival from the GEO database, suggesting that DLBCL patients with higher ORMDL1 levels tended to have lower OS (Figures 3(e) and 3(f)).

3.4. Genetic Alterations of ORMDL1 in DLBCL. Since ORMDL1 might play a role in DLBCL, genetic alterations of ORMDL1 in DLBCL were determined using cBioPortal database analysis. ORMDL1 mutations included gene gain and shallow deletion from 48 DLBCL patients (TCGA, Provisional) (Figure 4(a)). The relationship between ORMDL1 genetic alterations and DLBCL patient survival was further evaluated. The Kaplan-Meier survival analysis showed that cases with genetic alterations were associated with worse prognosis (Figure 4(b)).

3.5. Coexpressed Genes and Functional Analysis of ORMDL1 in DLBCL. To figure out the potential interaction of ORMDL1 with other genes in DLBCL, correlation analysis between $O R M D L 1$ and various genes and markers was performed via LinkedOmics. As shown in Figure 5, the top 50 significant genes positively and negatively correlated with ORMDL1 were shown in the heat map. A detailed description of the coexpression genes is listed in Table 1. Furthermore, Gene Ontology (GO) analysis in biological process by GSEA indicated that $O R M D L 1$ coexpressed genes mainly participated in DNA damage response, nucleus localization, rRNA metabolic process, and cell cycle checkpoint (Figure 6(a)). Kyoto Encyclopedia of Genes and Genomes (KEGG) 


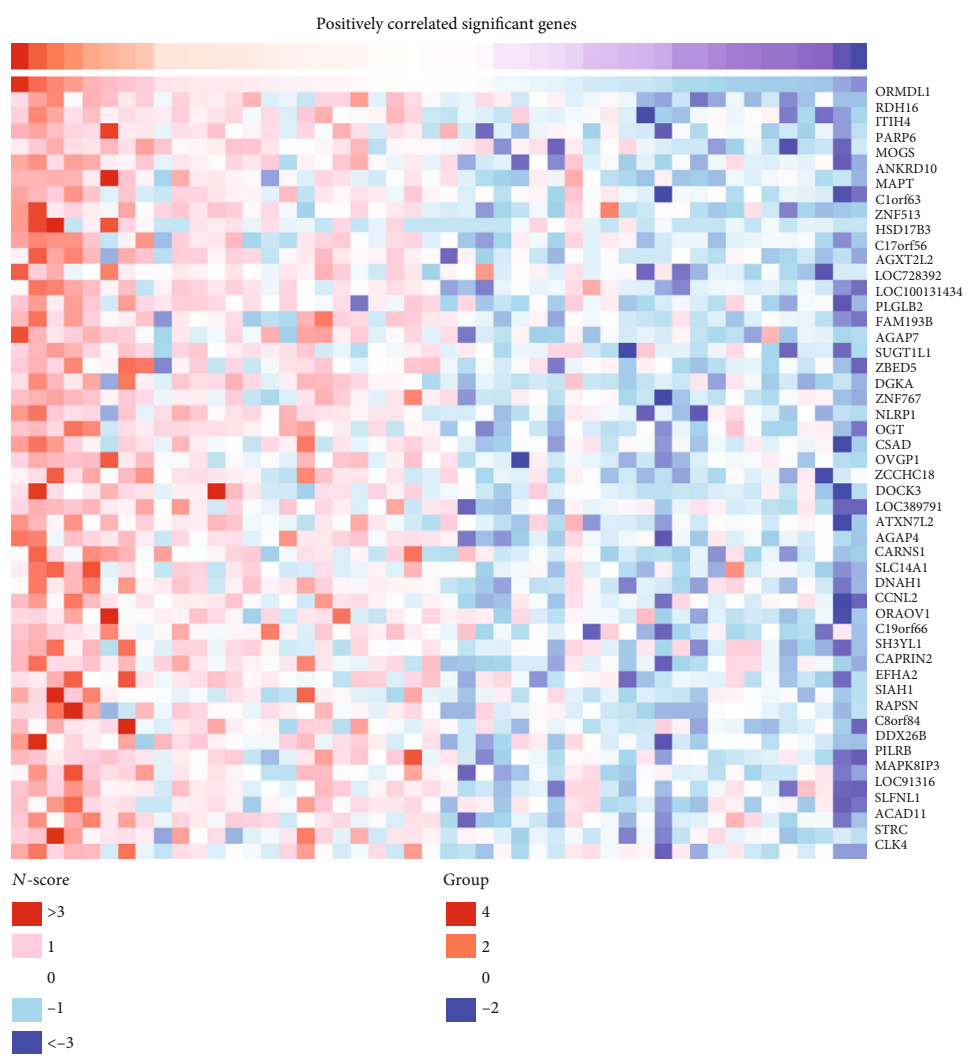

(a)
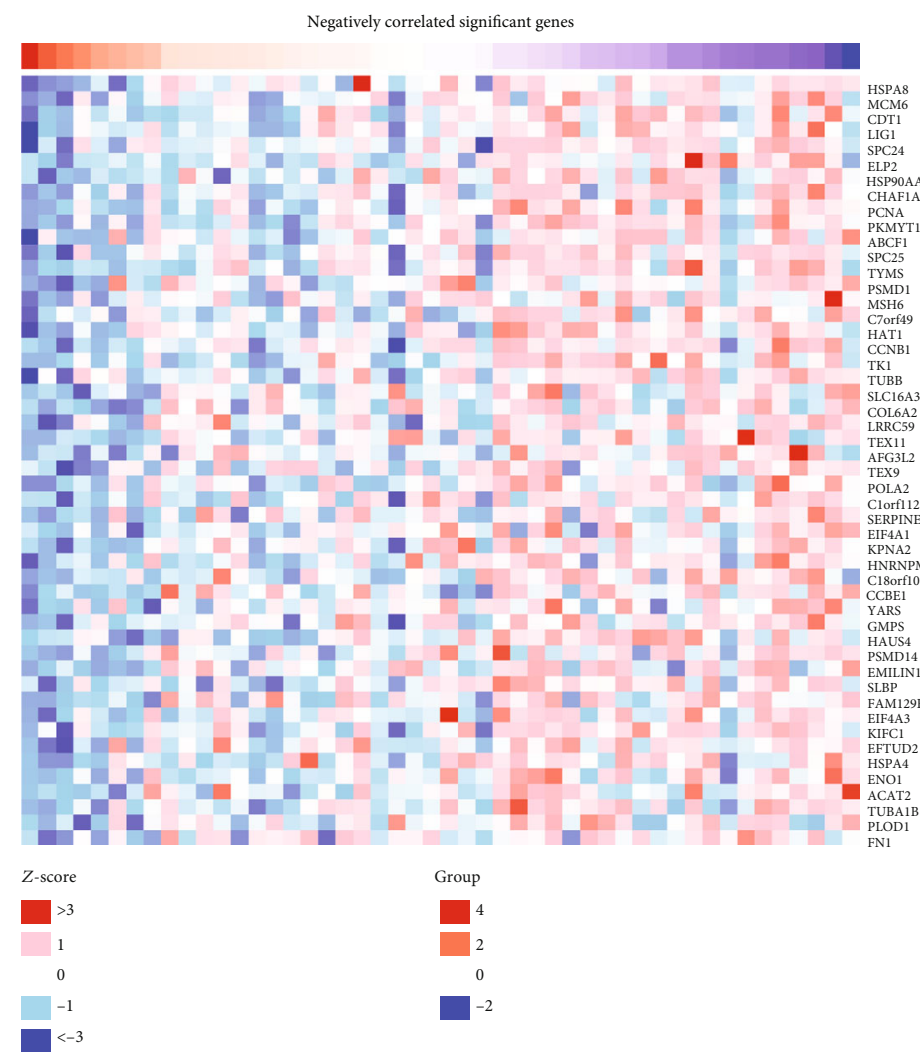

(b)

Figure 5: Coexpressed gene patterns of ORMDL1 in DLBCL (LinkedOmics). DLBCL: lymphoid neoplasm diffuse large B cell lymphoma. 
TABLE 1: Correlation analysis between ORMDL1 and related genes in DLBCL by LinkedOmics.

\begin{tabular}{lcc}
\hline Gene names & Pearson & DLBCL \\
\hline RBM6 & $3.736 e-01$ & $P$ value \\
ELMOD3 & $5.138 e-01$ & $8.902 e-03$ \\
ANKRD13D & $4.768 e-01$ & $1.876 e-04$ \\
C1orf63 & $6.451 e-01$ & $6.130 e-04$ \\
SFRS18 & $4.299 e-01$ & $7.428 e-07$ \\
STX16 & $4.791 e-01$ & $2.747 e-03$ \\
SUPT7L & $3.958 e-01$ & $5.704 e-04$ \\
DCUN1D2 & $3.992 e-01$ & $5.358 e-03$ \\
POLA2 & $-4.732 e-01$ & $4.943 e-03$ \\
SFRS17A & $4.085 e-01$ & $6.819 e-04$ \\
TMC8 & $4.460 e-01$ & $3.944 e-03$ \\
SORBS1 & $4.469 e-01$ & $1.486 e-03$ \\
PPWD1 & $4.299 e-01$ & $1.452 e-03$ \\
\hline
\end{tabular}

pathway analysis showed enrichment in cell cycle, ABC transporters, oxidative phosphorylation, and DNA replication (Figure 6(b)).

3.6. ORMDL1 Is Correlated with Immune Infiltration Level in $D L B C L$. To understand the relationship between ORMDL1 expression and immune signatures, we analyzed the six immune marker genes in DLBCL, including B cells, CD8+ $\mathrm{T}$ cells, CD4+ $\mathrm{T}$ cells, macrophages, neutrophils, and dendritic cells. The results revealed that the expression level of $O R M D L 1$ was significantly correlated with the infiltrating level of B cells in DLBCL (Figure 7(a)). Moreover, the gene gain mutation of ORMDL1 promoted the B cell infiltration in DLBCL (Figure 7(b)).

\section{Discussion}

Actually, the ORMDL gene family is a group of evolutionary conserved gene sequence found in Drosophila, yeast, and mammals. Among them, Drosophila only has a single-copy gene, yeast has two homologous genes of Orm1 and Orm2, and mammalian cells contain three homologous genes with ORMDL1, ORMDL2, and ORMDL3 [1]. The three human $O R M D L$ isoforms are located on chromosomes $2 \mathrm{q} 32$, $12 \mathrm{q} 13$, and $17 \mathrm{q} 21$, respectively, with approximately $80 \%$ identical amino acid, proving that they may have some common biological functions $[1,12,24]$. The most important function of ORMDLs is to regulate sphingolipid biosynthesis and maintain ceramide homeostasis [3-10, 25-28], where $\mathrm{SPT}$ is the rate-limiting enzyme. In yeast, the $\mathrm{Orm} / \mathrm{SPT}$ compound regulates sphingolipid expression levels by a negative feedback response. When the sphingolipid concentration is high, the Orm protein binds to SPT to inhibit SPT activity and reduce the further synthesis of sphingolipid. When the sphingolipid concentration is low, the N-terminal region phosphorylation of Orm protein causes its separation from
SPT, which eliminates the repression of SPT and promotes sphingolipid biosynthesis $[3,4,24,25]$. However, the regulation mechanism similar to that of yeast cannot be found in mammalian ORMDLs since human ORMDL proteins lack N-terminal phosphorylation sites $[1,4]$. The study by Siow and Wattenberg demonstrated the feedback response of ORMDL-mediated sphingolipid synthesis [10]. The phenomenon that inhibition of SPT activity caused by permeable cells treated with C6 ceramide suggested ORMDLs might have a structural domain interacting with C6 ceramide, which was further involved in the regulation of ORMDLdependent SPT activity. In addition, Wang et al. explored the relationship between ORMDL1, SPT, and sphingomyelin based on a free cholesterol- (FC-) loading microenvironment in human atherosclerotic macrophages [7, 9]. According to their research, the induction of endoplasmic reticulum (ER) stress and autophagy in FC-loaded macrophages led to ORMDL1 shifting from ER to autophagosome, followed by the dissociation of SPT, which was originally bound to ORMDL1. Then, the activation of SPT resulted in increased sphingomyelin synthesis, excessive FC buffering, and reduced cytotoxicity.

Dysregulation of sphingolipid metabolism in cancers has been described in several studies [29-35]. Typical sphingolipid metabolites such as ceramide and sphingosine could be used as bioactive signaling molecules, suppressing cell growth and promoting apoptosis [31]. Phosphorylated metabolites such as sphingosine-1-phosphate (S1P) are related with survival, proliferation, and migration of cancer cells $[36,37]$. The metabolism of bioactive sphingolipids in mammals is regulated by around 40 enzymes, which play key roles in cancer signaling pathways and therapeutic targets [29, 31, 32]. Consistently, sphingolipid enzymes and metabolites were abnormally expressed in a variety of cancers. For example, ceramide levels were upregulated in head and neck cancer and breast cancer $[38,39]$, while they were downregulated in ovarian cancer and colon cancer [40, 41]. Moreover, sphingosine was highly expressed in endometrial cancer [42]; S1P was overexpressed in glioblastoma [43], and SPT was lowly expressed in colon cancer [44]. Therefore, it was reconfirmed that metabolic disorders of sphingolipids interacted closely with tumorigenesis, tumor development, and chemoresistance of cancer patients.

As described above, ORMDLs act as critical factors in maintaining the balance of cellular sphingolipid levels. However, it remains unclear whether ORMDLs are involved in cancer networks associated with sphingolipid metabolism. In our study, we first preliminarily analyzed ORMDL1 expression in tumor tissues and normal tissues using the GEPIA database and found that ORMDL1 was expressed differently in diverse cancer tissues and adjacent tissues, especially highly expressed in CHOL, DLBCL, LAML, and THYM. Second, high expression of ORMDL1 in cell lines of DLBCL and LAML was verified by the CCLE database, which was consistent with the results in the corresponding tumor samples. To further elucidate the prognostic effect of ORMDL1 expression on CHOL, DLBCL, LAML, and THYM, the Kaplan-Meier survival curves were generated by GEPIA. It revealed that $O R M D L 1$ overexpression was significantly 
GO_BP

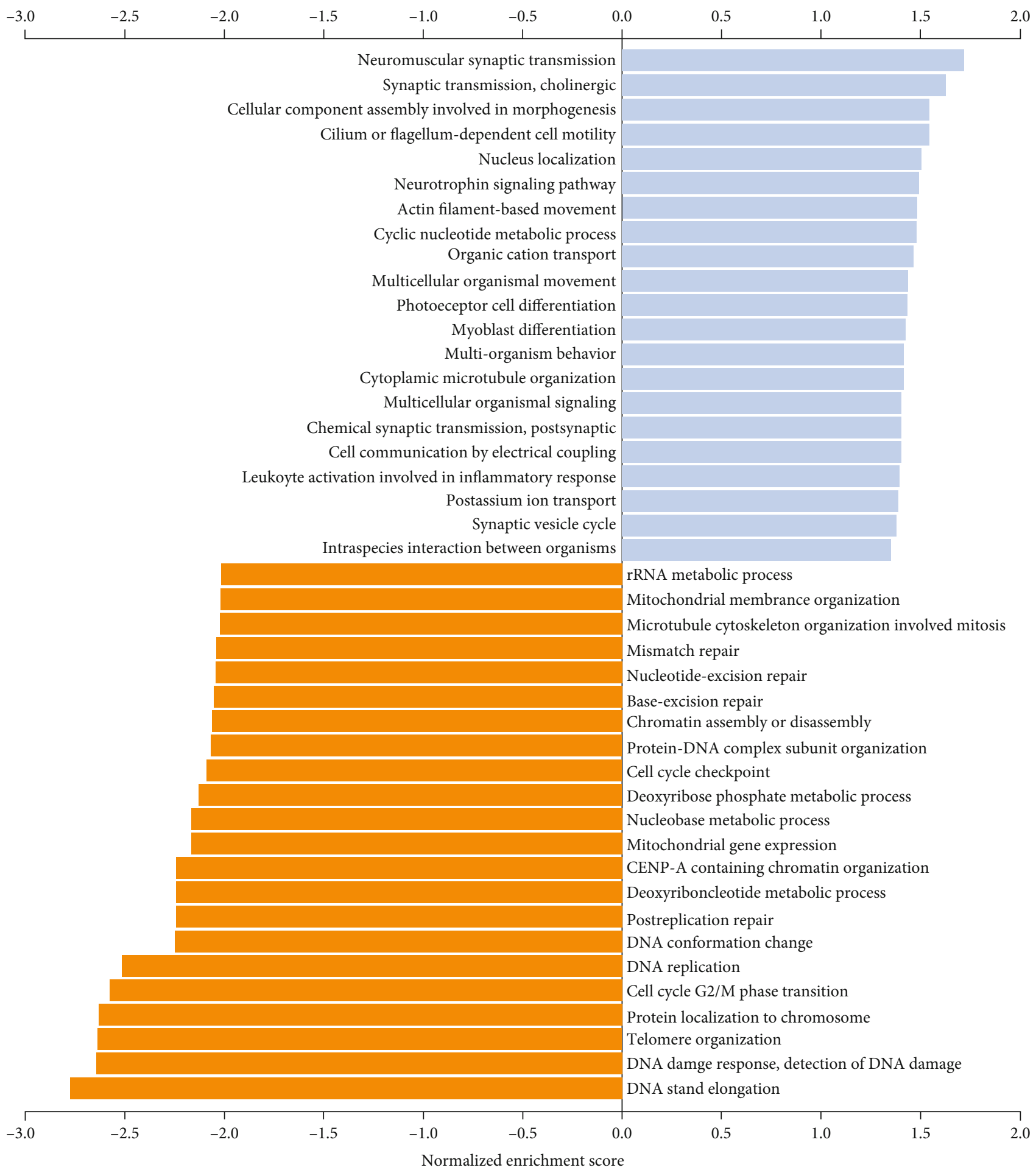

$\mathrm{FDR} \leq 0.05$

FDR $>0.05$

Figure 6: Continued. 


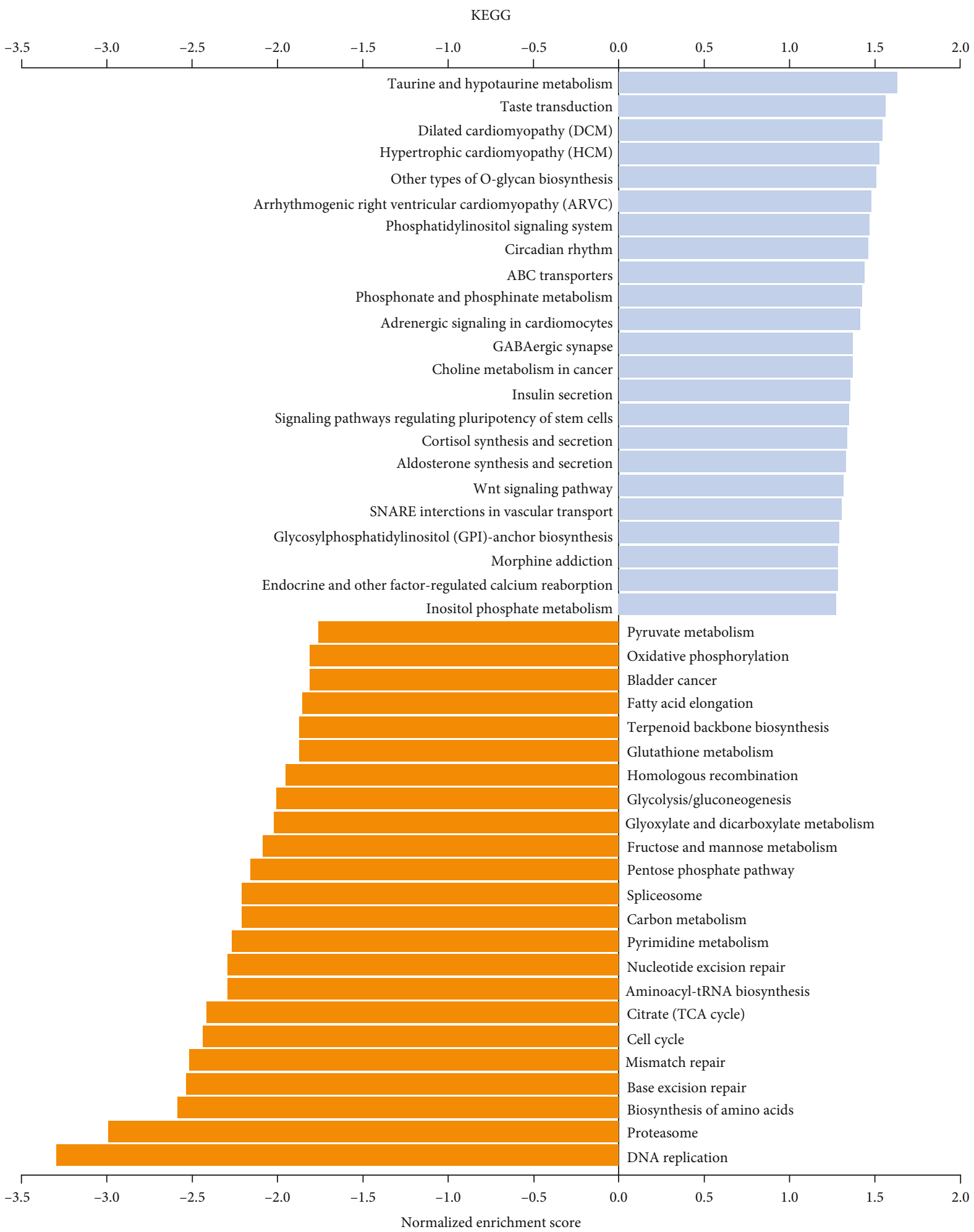

$\mathrm{FDR} \leq 0.05$

FDR $>0.05$

(b)

FIGURE 6: Functional analysis of ORMDL1 in DLBCL. (a) Gene Ontology (GO) analysis indicated ORMDL1 mainly participated in biological events like DNA damage response, nucleus localization, rRNA metabolic process, and cell cycle checkpoint. (b) Kyoto Encyclopedia of Genes and Genomes (KEGG) pathway analysis showed ORMDL1 enriched in cell cycle, ABC transporters, oxidative phosphorylation, and DNA replication. DLBCL: lymphoid neoplasm diffuse large B cell lymphoma. 


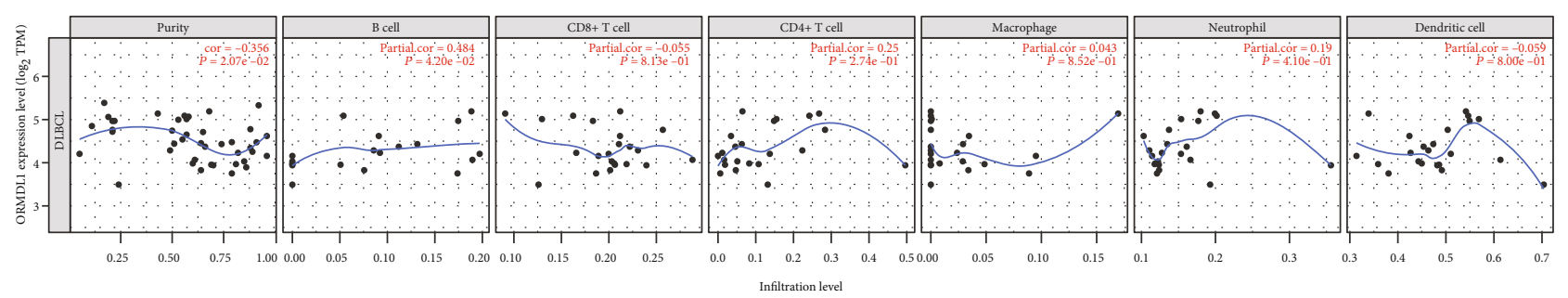

(a)

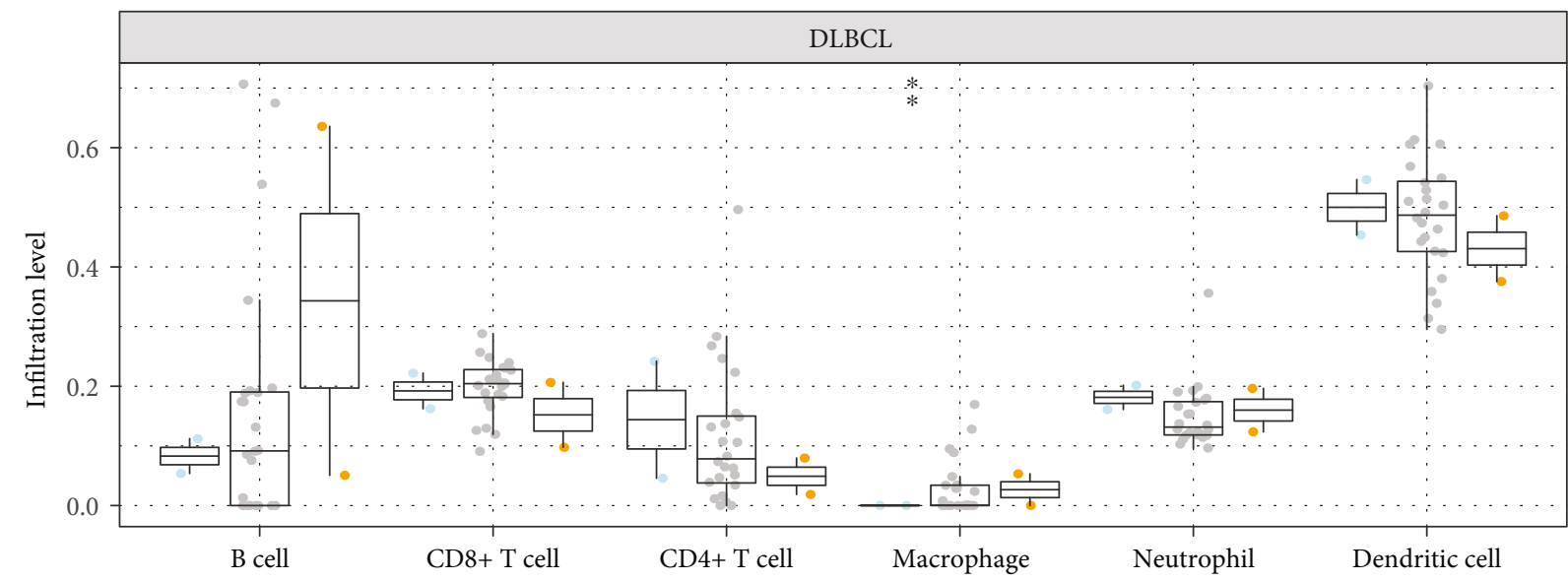

Copy number

Arm-level deletion

- Diploid/normal

审 Arm-level gain

(b)

FIGURE 7: The relationship between ORMDL1 expression and immune signatures. (a) The expression level of ORMDL1 was significantly correlated with the infiltrating level of B cells in DLBCL. (b) The gene gain mutation of ORMDL1 promoted the B cell infiltration in DLBCL. DLBCL: lymphoid neoplasm diffuse large B cell lymphoma.

associated with poor survival of DLBCL, indicating ORMDL1 might facilitate tumorigenesis and recurrence in DLBCL. The results of GSE10846 and GSE53786 further confirmed the results that high expression of ORMDL1 indicated poor prognosis in DLBCL patients. In addition, the cBioPortal database was used as a powerful tool for discovering ORMDL1 genetic alterations in DLBCL, since genetic alteration was considered as an important factor in cancer development $[45,46]$. Expectedly, increased gene copies and slight gene deletion existed in DLBCL, which partly explained the higher expression of ORMDL1 in DLBCL compared with normal samples. The concomitant result also showed cases with ORMDL1 genetic alterations had worse prognosis. Finally, through LinkedOmics and Pearson correlation test, genes that positively and negatively interacted with ORMDL1 were found and functional analysis in GO and KEGG pathways was further explored, which might be jointly involved in the ORMDL1-related cancer signaling pathways.

There were still some limitations to be solved. Firstly, differences of sample sizes among multidatabases might cause some bias. Secondly, this study only analyzed transcriptional levels of ORMDL1 in cancers, without its posttranslational levels. Finally, molecular mechanism investigation should be carried out to further explore can- cer pathways associated with ORMDL1 and sphingolipid metabolism.

\section{Conclusions}

This was the initial study comprehensively analyzing the expression patterns and prognostic values of ORMDL1 in different tumors. ORMDL1 is promising to be the potential therapeutic target and prognostic marker in DLBCL.

\section{Data Availability}

Data can be available upon request.

\section{Conflicts of Interest}

All authors have no conflicts of interest to declare.

\section{Authors' Contributions}

Dr. Tengjiao Zhu and Dr. Yingtong Chen contributed equally to this article in the aspects of drafting the work as well as acquiring and interpreting the data for the work. Dr. Shuyuan Min was accountable for the statistics work of the article. Dr. Li and Dr. Yun Tian made substantial 
contributions to the conception and design of the work. Tengjiao Zhu and Yingtong Chen contributed equally to this article.

\section{Acknowledgments}

This work was supported by the grants from the Capital Clinical Characteristic Application Research Project (Z181100001718195).

\section{References}

[1] L. Hjelmqvist, M. Tuson, G. Marfany, E. Herrero, S. Balcells, and R. Gonzalez-Duarte, "ORMDL proteins are a conserved new family of endoplasmic reticulum membrane proteins," Genome Biology, vol. 3, no. 6, 2002.

[2] W. Araki, N. Takahashi-Sasaki, D. H. Chui et al., "A family of membrane proteins associated with presenilin expression and gamma-secretase function," FASEB Journal : Official Publication of the Federation of American Societies for Experimental Biology, vol. 22, no. 3, pp. 819-827, 2008.

[3] S. Han, M. A. Lone, R. Schneiter, and A. Chang, "Orm1 and Orm2 are conserved endoplasmic reticulum membrane proteins regulating lipid homeostasis and protein quality control," Proceedings of the National Academy of Sciences of the United States of America, vol. 107, no. 13, pp. 5851-5856, 2010.

[4] D. K. Breslow, S. R. Collins, B. Bodenmiller et al., "Orm family proteins mediate sphingolipid homeostasis," Nature, vol. 463, no. 7284, pp. 1048-1053, 2010.

[5] K. Kiefer, A. Carreras-Sureda, R. García-López et al., “Coordinated regulation of the orosomucoid-like gene family expression controls de novo ceramide synthesis in mammalian cells," The Journal of Biological Chemistry, vol. 290, no. 5, pp. 2822-2830, 2015.

[6] S. D. Gupta, K. Gable, A. Alexaki et al., "Expression of the ORMDLS, modulators of serine palmitoyltransferase, is regulated by sphingolipids in mammalian cells," The Journal of Biological Chemistry, vol. 290, no. 1, pp. 90-98, 2015.

[7] S. Wang, P. Robinet, J. D. Smith, and K. Gulshan, "Free-cholesterol-mediated autophagy of ORMDL1 stimulates sphingomyelin biosynthesis," Autophagy, vol. 11, no. 7, pp. 1207-1208, 2015.

[8] L. Cai, C. Oyeniran, D. D. Biswas et al., "ORMDL proteins regulate ceramide levels during sterile inflammation," Journal of Lipid Research, vol. 57, no. 8, pp. 1412-1422, 2016.

[9] S. Wang, P. Robinet, J. D. Smith, and K. Gulshan, "ORMDL orosomucoid-like proteins are degraded by free-cholesterolloading-induced autophagy," Proceedings of the National Academy of Sciences of the United States of America, vol. 112, no. 12 , pp. 3728-3733, 2015.

[10] D. L. Siow and B. W. Wattenberg, "Mammalian ORMDL proteins mediate the feedback response in ceramide biosynthesis," The Journal of Biological Chemistry, vol. 287, no. 48, pp. 40198-40204, 2012.

[11] M. F. Moffatt, M. Kabesch, L. Liang et al., "Genetic variants regulating ORMDL3 expression contribute to the risk of childhood asthma," Nature, vol. 448, no. 7152, pp. 470-473, 2007.

[12] A. A. Toncheva, D. P. Potaczek, M. Schedel et al., "Childhood asthma is associated with mutations and gene expression differences of ORMDL genes that can interact," Allergy, vol. 70, no. 10, pp. 1288-1299, 2015.
[13] G. Cantero-Recasens, C. Fandos, F. Rubio-Moscardo, M. A. Valverde, and R. Vicente, "The asthma-associated ORMDL3 gene product regulates endoplasmic reticulum-mediated calcium signaling and cellular stress," Human Molecular Genetics, vol. 19, no. 1, pp. 111-121, 2010.

[14] A. Carreras-Sureda, G. Cantero-Recasens, F. Rubio-Moscardo et al., "ORMDL3 modulates store-operated calcium entry and lymphocyte activation," Human Molecular Genetics, vol. 22, no. 3, pp. 519-530, 2013.

[15] S. G. Ha, X. N. Ge, N. S. Bahaie et al., "ORMDL3 promotes eosinophil trafficking and activation via regulation of integrins and CD48," Nature Communications, vol. 4, no. 1, p. 2479, 2013.

[16] Y. A. Hannun and L. M. Obeid, "Principles of bioactive lipid signalling: lessons from sphingolipids," Nature Reviews Molecular Cell Biology, vol. 9, no. 2, pp. 139-150, 2008.

[17] Y. A. Hannun and R. M. Bell, "Lysosphingolipids inhibit protein kinase C: implications for the sphingolipidoses," Science, vol. 235, no. 4789, pp. 670-674, 1987.

[18] K. A. Dressler, S. Mathias, and R. N. Kolesnick, “Tumor necrosis factor-alpha activates the sphingomyelin signal transduction pathway in a cell-free system," Science, vol. 255, no. 5052, pp. 1715-1718, 1992.

[19] Z. Tang, C. Li, B. Kang, G. Gao, C. Li, and Z. Zhang, "GEPIA: a web server for cancer and normal gene expression profiling and interactive analyses," Nucleic Acids Research, vol. 45, no. W1, pp. W98-w102, 2017.

[20] D. Sonkin, M. Hassan, D. J. Murphy, and T. V. Tatarinova, "Tumor suppressors status in Cancer Cell Line Encyclopedia," Molecular Oncology, vol. 7, no. 4, pp. 791-798, 2013.

[21] S. V. Vasaikar, P. Straub, J. Wang, and B. Zhang, "LinkedOmics: analyzing multi-omics data within and across 32 cancer types," Nucleic Acids Research, vol. 46, no. D1, pp. D956-d963, 2018.

[22] J. Gao, B. A. Aksoy, U. Dogrusoz et al., "Integrative analysis of complex cancer genomics and clinical profiles using the cBioPortal," Science Signaling, vol. 6, no. 269, article pl1, 2013.

[23] B. Li, E. Severson, J. C. Pignon et al., "Comprehensive analyses of tumor immunity: implications for cancer immunotherapy," Genome Biology, vol. 17, no. 1, p. 174, 2016.

[24] D. Davis, M. Kannan, and B. Wattenberg, "Orm/ORMDL proteins: gate guardians and master regulators," Advances in Biological Regulation, vol. 70, pp. 3-18, 2018.

[25] F. M. Roelants, D. K. Breslow, A. Muir, J. S. Weissman, and J. Thorner, "Protein kinase Ypk1 phosphorylates regulatory proteins Orm1 and Orm2 to control sphingolipid homeostasis in Saccharomyces cerevisiae," Proceedings of the National Academy of Sciences of the United States of America, vol. 108, no. 48, pp. 19222-19227, 2011.

[26] M. Liu, C. Huang, S. R. Polu, R. Schneiter, and A. Chang, "Regulation of sphingolipid synthesis through Orm1 and Orm2 in yeast," Journal of Cell Science, vol. 125, no. 10, pp. 2428-2435, 2012.

[27] M. Shimobayashi, W. Oppliger, S. Moes, P. Jeno, and M. N. Hall, "TORC1-regulated protein kinase Npr1 phosphorylates Orm to stimulate complex sphingolipid synthesis," Molecular Biology of the Cell, vol. 24, no. 6, pp. 870-881, 2013.

[28] C. Gururaj, R. S. Federman, and A. Chang, "Orm proteins integrate multiple signals to maintain sphingolipid homeostasis," The Journal of Biological Chemistry, vol. 288, no. 28, pp. 20453-20463, 2013. 
[29] B. Ogretmen, "Sphingolipid metabolism in cancer signalling and therapy," Nature Reviews Cancer, vol. 18, no. 1, pp. 3350, 2018.

[30] J. Shaw, P. Costa-Pinheiro, L. Patterson, K. Drews, S. Spiegel, and M. Kester, "Novel sphingolipid-based cancer therapeutics in the personalized medicine era," Advances in Cancer Research, vol. 140, pp. 327-366, 2018.

[31] L. K. Ryland, T. E. Fox, X. Liu, T. P. Loughran, and M. Kester, "Dysregulation of sphingolipid metabolism in cancer," Cancer Biology \& Therapy, vol. 11, no. 2, pp. 138-149, 2011.

[32] Y. A. Hannun and L. M. Obeid, "Sphingolipids and their metabolism in physiology and disease," Nature Reviews Molecular Cell Biology, vol. 19, no. 3, pp. 175-191, 2018.

[33] W. K. Lee and R. N. Kolesnick, "Sphingolipid abnormalities in cancer multidrug resistance: chicken or egg?," Cellular Signalling, vol. 38, pp. 134-145, 2017.

[34] S. A. F. Morad and M. C. Cabot, "Ceramide-orchestrated signalling in cancer cells," Nature Reviews Cancer, vol. 13, no. 1, pp. 51-65, 2013.

[35] J. Newton, S. Lima, M. Maceyka, and S. Spiegel, "Revisiting the sphingolipid rheostat: evolving concepts in cancer therapy," Experimental Cell Research, vol. 333, no. 2, pp. 195-200, 2015.

[36] V. E. Nava, J. P. Hobson, S. Murthy, S. Milstien, and S. Spiegel, "Sphingosine kinase type 1 promotes estrogen-dependent tumorigenesis of breast cancer MCF-7 cells," Experimental Cell Research, vol. 281, no. 1, pp. 115-127, 2002.

[37] F. Wang, J. R. Van Brocklyn, L. Edsall, V. E. Nava, and S. Spiegel, "Sphingosine-1-phosphate inhibits motility of human breast cancer cells independently of cell surface receptors," Cancer Research, vol. 59, no. 24, pp. 6185-6191, 1999.

[38] S. Karahatay, K. Thomas, S. Koybasi et al., "Clinical relevance of ceramide metabolism in the pathogenesis of human head and neck squamous cell carcinoma (HNSCC): attenuation of $\mathrm{C}(18)$-ceramide in HNSCC tumors correlates with lymphovascular invasion and nodal metastasis," Cancer Letters, vol. 256, no. 1, pp. 101-111, 2007.

[39] S. Schiffmann, J. Sandner, K. Birod et al., "Ceramide synthases and ceramide levels are increased in breast cancer tissue," Carcinogenesis, vol. 30, no. 5, pp. 745-752, 2009.

[40] S. N. Rylova, O. G. Somova, and E. V. Dyatlovitskaya, "Comparative investigation of sphingoid bases and fatty acids in ceramides and sphingomyelins from human ovarian malignant tumors and normal ovary," Biochemistry Biokhimiia, vol. 63, no. 9, pp. 1057-1060, 1998.

[41] M. Selzner, A. Bielawska, M. A. Morse et al., "Induction of apoptotic cell death and prevention of tumor growth by ceramide analogues in metastatic human colon cancer," Cancer Research, vol. 61, no. 3, pp. 1233-1240, 2001.

[42] P. Knapp, M. Baranowski, M. Knapp, P. Zabielski, A. U. Blachnio-Zabielska, and J. Gorski, "Altered sphingolipid metabolism in human endometrial cancer," Prostaglandins \& Other Lipid Mediators, vol. 92, no. 1-4, pp. 62-66, 2010.

[43] J. R. Van Brocklyn, C. A. Jackson, D. K. Pearl, M. S. Kotur, P. J. Snyder, and T. W. Prior, "Sphingosine kinase-1 expression correlates with poor survival of patients with glioblastoma multiforme: roles of sphingosine kinase isoforms in growth of glioblastoma cell lines," Journal of Neuropathology and Experimental Neurology, vol. 64, no. 8, pp. 695-705, 2005.

[44] R. Duan and A. Nilsson, "Metabolism of sphingolipids in the gut and its relation to inflammation and cancer development," Progress in Lipid Research, vol. 48, no. 1, pp. 62-72, 2009.
[45] C. C. Sun, Q. Zhou, W. Hu et al., "Transcriptional E2F1/2/5/8 as potential targets and transcriptional E2F3/6/7 as new biomarkers for the prognosis of human lung carcinoma," Aging, vol. 10, no. 5, pp. 973-987, 2018.

[46] J. Mei, L. Hao, X. Liu et al., "Comprehensive analysis of peroxiredoxins expression profiles and prognostic values in breast cancer," Biomarker Research, vol. 7, no. 1, p. 16, 2019. 